\title{
Synthesis and chemical etching of Te/C nanocables
}

\author{
GUANG SHENG CAO*, YONG GANG LIU, WEN WU YANG, CHANG TAN, HUI LI \\ and XIAO JUAN ZHANG \\ Key Laboratory of Enhanced Oil \& Gas Recovery of Ministry of Education, Northeast Petroleum University, \\ Daqing 163318, P. R. China
}

MS received 31 December 2010; revised 5 April 2011

\begin{abstract}
In this paper, Te/C nanocables were fabricated by a hydrothermal method in the presence of cetyltrimethylammonium bromide (CTAB). The products were characterized in detail by multiform techniques: transmission electron microscopy, $\mathrm{X}$-ray diffraction, energy-dispersive $\mathrm{X}$-ray analysis and Fourier transform infrared (FTIR) spectroscopy. The results showed that the products were nanocables with lengths of several microns, core about $20 \mathrm{~nm}$ in diameter, and a surrounding sheath of about $60-80 \mathrm{~nm}$ in thickness. Te/C nanocables were tailored freely by chemical etching. Carbonaceous nanotubes and $\mathrm{Te} / \mathrm{C}$ nanocables with fragmentary Te core were obtained by adjusting time of chemical etching.
\end{abstract}

Keywords. Carbon; Te nanocable; heterostructure; chemical etching.

\section{Introduction}

Tellurium (Te) is a narrow bandgap semiconductor $(\sim 0.35 \mathrm{eV})$ that exhibits a wealth of unique useful properties, such as nonlinear optical response, photoconductivity and thermoelectric properties. These properties make it useful as an infrared photoconductive detector, piezoelectronic device, and thermoelectronic material (Xi et al 2005; Song et al 2009). The size and shape of nanostructures are two crucial factors in determining the properties of nanomaterials, and thus, the control of size and shape is of great interest (Lu et al 2005; Song et al 2007a). Recently, many methods have been developed to fabricate Te nanostructures. Mo et al (2002) synthesized $\mathrm{Te}$ nanobelts by the in situ disproportionation of sodium tellurite $\left(\mathrm{Na}_{2} \mathrm{TeO}_{3}\right)$ in an aqueous ammonia system at $180^{\circ} \mathrm{C}$. Mayers and Xia (2002) reported solutionphase synthesis approaches of tellurium nanotubes. Liu et al (2003, 2004) synthesized Te nanorods and nanowires by a $\mathrm{Na}_{2} \mathrm{SO}_{3}$ reducing $\left(\mathrm{NH}_{4}\right)_{2} \mathrm{TeS}_{4}$ route. Using biomolecules as structure-directing agents, $\mathrm{He}$ and $\mathrm{Yu}$ (2005) synthesized scrolled Te nanotubes.

Nanocable, a new kind of 1-D nanocomposite of nanowires (core) wrapped with one or more outer layers (shell), has emerged recently and drawn much intensive investigations. Nanocables reveal some fancy performances for their core-shell structure, so they can be used as the connection wires among high-density integrated elements, super capacitor, micro-implements and elements of microrobot. A number of approaches, such as thermal evaporation, $\gamma$-irradiation, and templating, have been developed

\footnotetext{
*Author for correspondence (daqingcgs@163.com)
}

to fabricate these kinds of 1-D nanocomposites (Xie et al 1999; Hu et al 2003; Jiang et al 2003). Iijima and his coworkers prepared coaxial three-layer nanostructures consisting of silicon carbide cores via a laser ablation route (Zhang et al 1997). Fang et al (2006) reported a CTAB-assisted hydrothermal approach to fabricate core-shell nanoparticles and nanocables with silver cores. Song et al (2008a) successfully prepared Se/C coaxial nanocables via one-step reduction and carbonization under mild hydrothermal condition. Zhang et al (2001) synthesized coaxial $\mathrm{TiO}_{2} / \mathrm{SiO}_{2}$ nanocables by using a sol-gel method with anodic alumina templates. Recently, a new method to prepare $\mathrm{Ag} / \mathrm{C}$ nanocables with cross-linked structure via SDS-assisted hydrothermal route was reported by Song et al (2008b). In this article, we present a simple hydrothermal route for the synthesis of Te/C nanocables by using glucose as reductant and $\mathrm{Na}_{2} \mathrm{TeO}_{3}$ as tellurium source. Using the as-prepared $\mathrm{Te} / \mathrm{C}$ nanocables as raw material, carbonaceous nanotubes and $\mathrm{Te} / \mathrm{C}$ nanocables with fragmentary Te cores were obtained by adjusting time of chemical etching.

\section{Experimental}

\subsection{Synthesis of Te/C nanocables}

All chemicals were of analytical grade without further purification. Experimental details are as follows: CTAB (1 g) was dissolved in $25 \mathrm{~mL}$ of distilled water, followed by addition of glucose $(2 \mathrm{~g})$ under vigorous stirring. When the solution attained clarity, $10 \mathrm{~mL}$ of aqueous solution containing $0.1 \mathrm{~g}$ of $\mathrm{Na}_{2} \mathrm{TeO}_{3}$ was added slowly to the above solution under continuous stirring. The final solution was transferred into a 

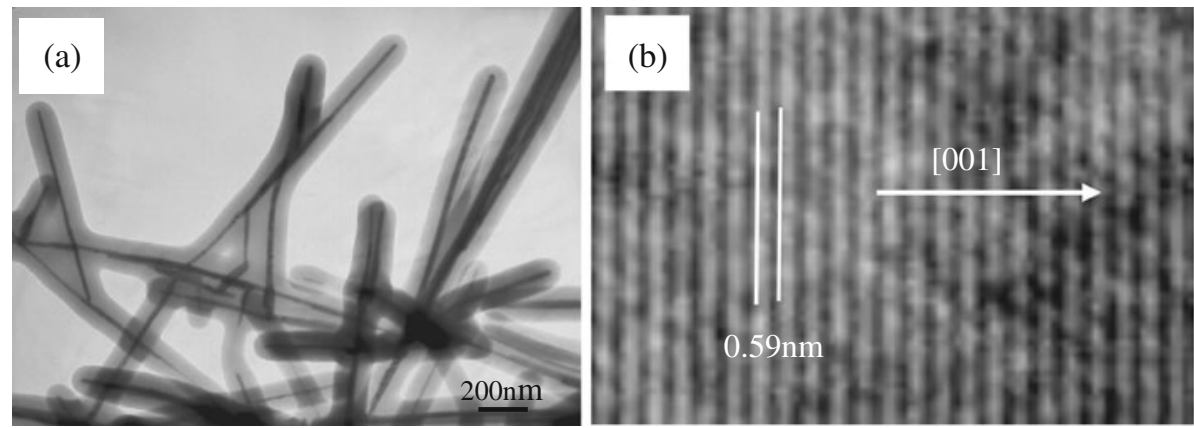

Figure 1. (a) TEM and (b) HRTEM of Te/C nanocables.

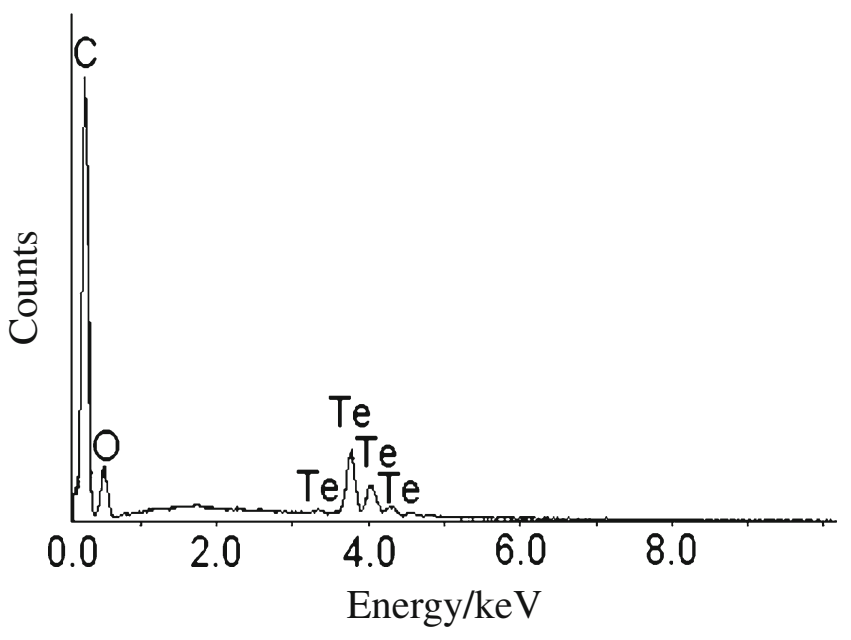

Figure 2. EDS spectrum of $\mathrm{Te} / \mathrm{C}$ nanocables.

$50 \mathrm{~mL}$ Teflon-lined stainless steel autoclave, and filled with distilled water up to $90 \%$ of the total volume. The autoclave was sealed and maintained at $190^{\circ} \mathrm{C}$ for $12 \mathrm{~h}$. After the reactions, the autoclave was allowed to cool to room temperature naturally. Finally, the products were collected and washed with deionized water and ethanol several times and dried in air at $80^{\circ} \mathrm{C}$.

\subsection{Chemical etching of Te/C nanocables}

Te/C nanocables $(0.1 \mathrm{~g})$ were dispersed into $25 \mathrm{~mL}$ of aqueous solution containing $2 \mathrm{~mL}$ hydrochloric acid (36.5 wt\%) and $5 \mathrm{~mL} \mathrm{H}_{2} \mathrm{O}_{2}(30 \mathrm{wt} \%)$. The above solution was kept still for 45-90 $\mathrm{min}$, and then was filtered to separate the precipitates. The precipitates were washed by distilled water and anhydrous ethanol several times. Then the products were dried at $80^{\circ} \mathrm{C}$ for further characterization.

\subsection{Characterization}

The morphologies of the products were characterized with transmission electron microscopy (TEM, JEM200CX, $120 \mathrm{kV}$ ). High-resolution TEM study was carried out with a JEOL

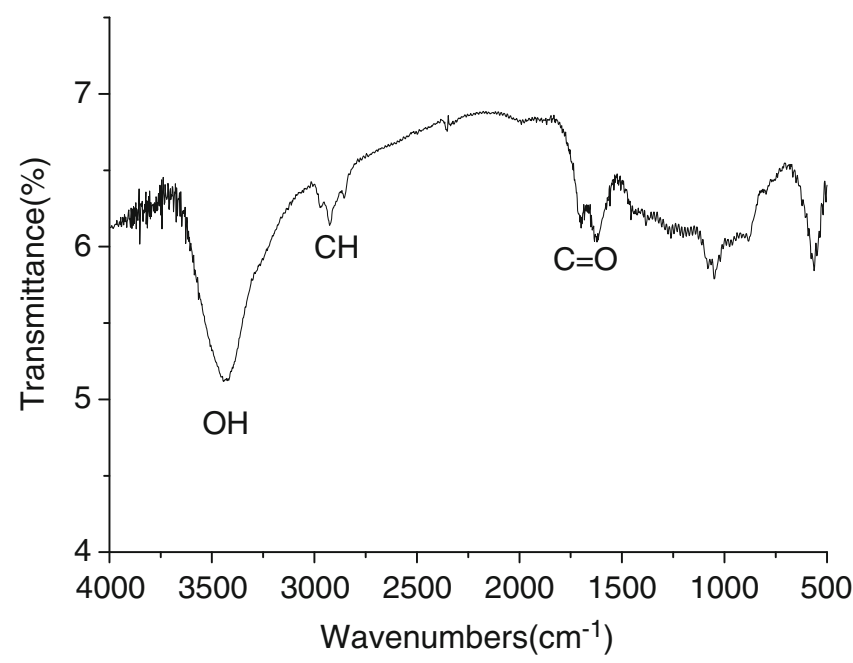

Figure 3. FTIR spectra of $\mathrm{Te} / \mathrm{C}$ nanocables.

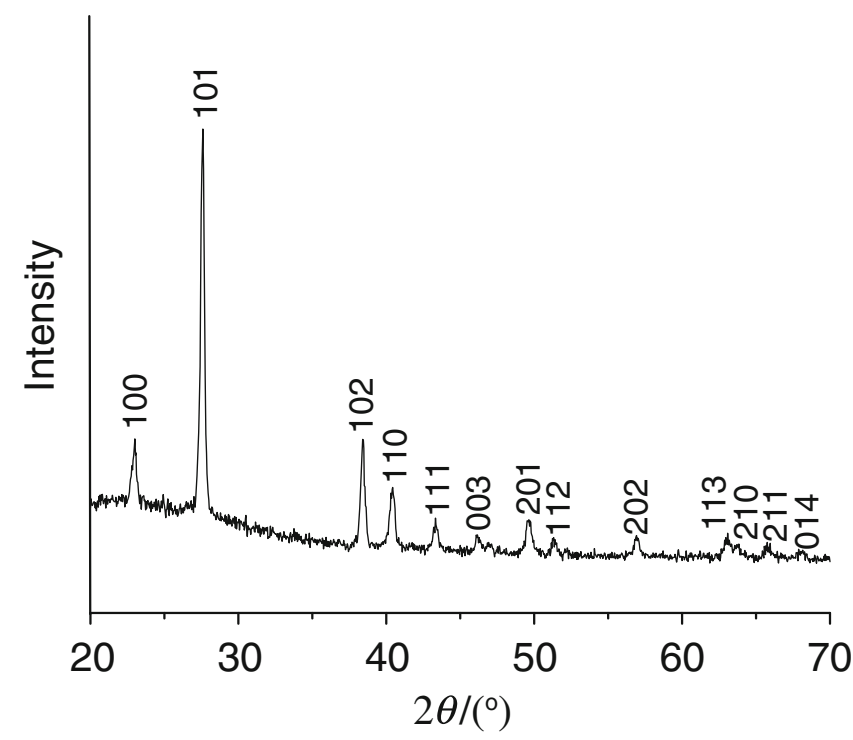

Figure 4. XRD pattern of $\mathrm{Te} / \mathrm{C}$ nanocables.

2010 microscope at an accelerating voltage of $200 \mathrm{kV}$. The composition of the products was analysed by energy dispersive X-ray detector (EDS, Thermo Noran VANTAG-ESI, 
$120 \mathrm{kV}$ ). The X-ray diffraction (XRD, Thermo ARL SCINTAG X'TRA with $\mathrm{CuK} \alpha$ irradiation, $\lambda=0.154056 \mathrm{~nm}$ ) was used to analyse the crystallinity of the products. IR measurement

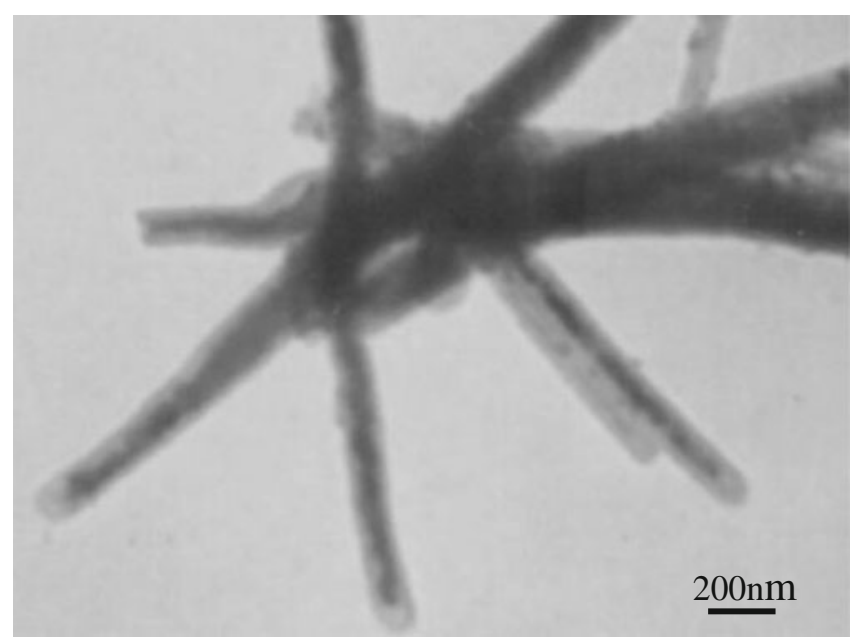

Figure 5. TEM image of sample obtained without CTAB.

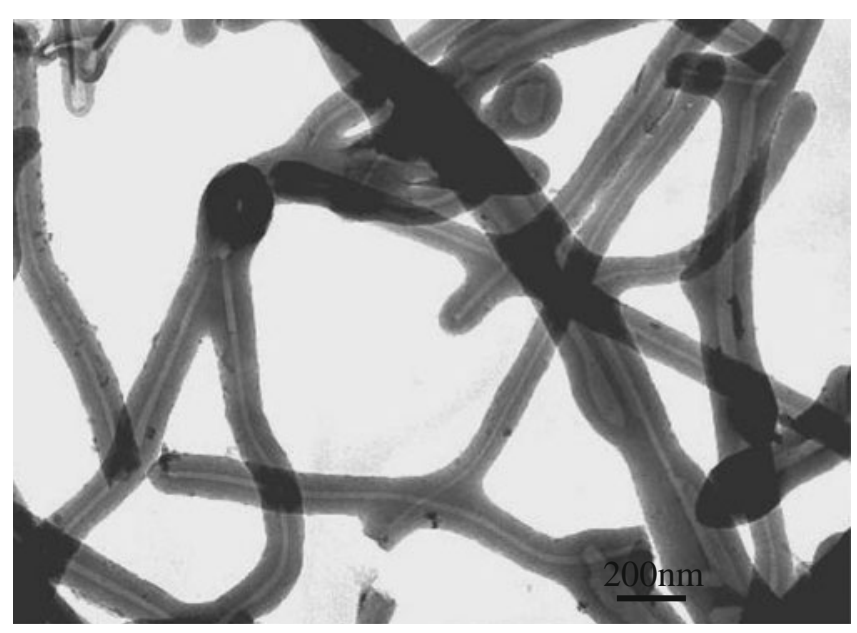

Figure 6. TEM image of carbonaceous nanotubes obtained by chemical etching of $\mathrm{Te} / \mathrm{C}$ nanocables. was carried out with a Nicolet Avatar 360 Fourier-transform infrared (FTIR) spectrophotometer.

\section{Results and discussion}

The morphologies and particle sizes of the samples were investigated by TEM. Figure 1a shows typical pattern of $\mathrm{Te} / \mathrm{C}$ nanocables prepared via CTAB-assisted hydrothermal route at $190^{\circ} \mathrm{C}$. TEM image shows that the products are a composite comprising of a smooth core of about $20 \mathrm{~nm}$ in diameter and a surrounding sheath of about $60-80 \mathrm{~nm}$ in thickness. HRTEM analysis provides more detailed structural information about Te core. Figure 1b is a HRTEM image taken from Te core. The observed interplanar spacing is $0.59 \mathrm{~nm}$, which corresponds to the separation between [001] planes of Te. And this HRTEM image indicates that the Te core growth direction is along [001]. The EDS analysis was employed to determine composition of the products and is shown in figure 2. The EDS results confirm that the obtained nanocables composed of inner tellurium nanowires and outer carbonaceous layers.

The FTIR spectra have been used to detect the functional groups of sheaths, as shown in figure 3 . The peaks around $3419 \mathrm{~cm}^{-1}, 2900 \mathrm{~cm}^{-1}$ and $1620 \mathrm{~cm}^{-1}$ are attributed to the $\mathrm{O}-\mathrm{H}, \mathrm{C}-\mathrm{H}$ and $\mathrm{C}=\mathrm{O}$ vibrations, respectively. The results show that the surface of these carbonaceous sheaths is obviously highly functionalized.

The phase and purity of the products were examined by $\mathrm{XRD}$. Figure 4 displays XRD patterns of the as-prepared $\mathrm{Te} / \mathrm{C}$ nanocables in the presence of $\mathrm{CTAB}$ at $190^{\circ} \mathrm{C}$. All the diffraction peaks can be indexed to be the trigonal phase of tellurium with lattice parameters $a=4.460 \AA$ and $c=$ $5.923 \AA$, which correspond with the values in the literature (JCPDS card No. 36-1452, $a=4.458 \AA$ and $c=5.927 \AA$ ). No other phases were detected in figure 4 , which indicates that high purity of $\mathrm{Te} / \mathrm{C}$ nanocables were obtained by the present synthetic method.

Figure 5 is the TEM image of the samples obtained without $\mathrm{CTAB}$ at $190^{\circ} \mathrm{C}$. The results show that the obtained products were nanocables with crude Te core. As we know, the crystal structure of tellurium is highly anisotropic, and the

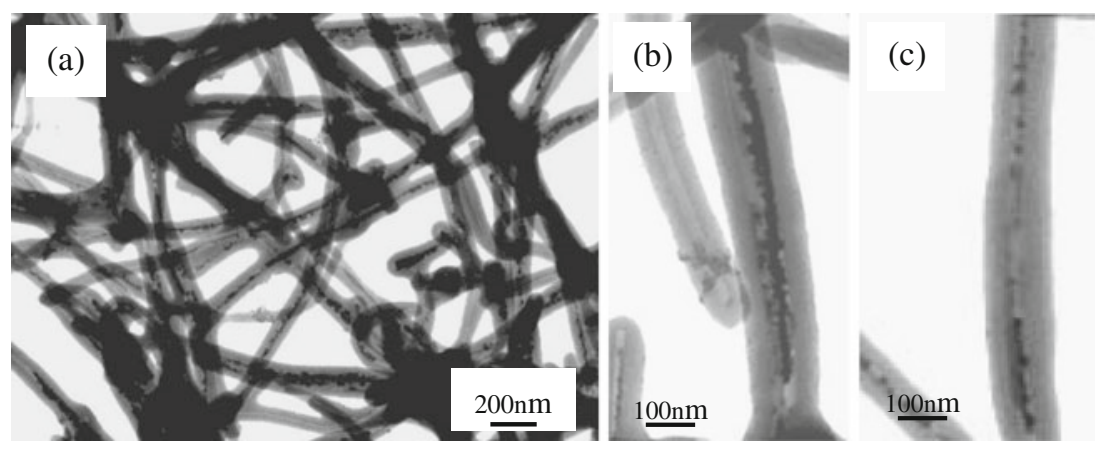

Figure 7. TEM images of $\mathrm{Te} / \mathrm{C}$ nanocables by partial chemical etching. 
formation of 1D nanostructure first needs anisotropy during the growing process of nanoparticles. The selective interaction of the capping molecules on the facets of the first-formed nanoparticles is crucial to the anisotropic growth of nanostructures (Song et al 2007b). According to the results, a possible formation mechanism of Te nanocables has been proposed. In our experiments, the presence of CTAB would help to enhance the anisotropy of tellurium and cause the formation of uniform 1-D structures. As is known to all, glucose is a typical soft reducer. The glucose solution is heated in the autoclaves to $160-180^{\circ} \mathrm{C}$, which is higher than the normal glycosidation temperature, and will lead to aromatization and carbonization. Based on above theories, we can conjecture that the formation process of $\mathrm{Te} / \mathrm{C}$ nanocables may undergo the following procedures: first, $\mathrm{TeO}_{3}^{2-}$ is deoxidized by glucose with the assistance of CTAB, and then Te nanowires core forms at earliest; along with hydrothermal reaction continuing, glucose in the system carbonizes to amorphous carbon, and an amorphous carbon layer coats on Te nanowires surface and results in the formation of $\mathrm{Te} / \mathrm{C}$ nanocables.

Figure 6 shows the TEM images of the products obtained after chemical etching for $90 \mathrm{~min}$. Large numbers of amorphous carbonaceous nanotubes can be obtained from $\mathrm{Te} / \mathrm{C}$ nanocables. Te can be dissolved by the solution of $\mathrm{H}_{2} \mathrm{O}_{2}$ and form $\mathrm{TeO}_{3}^{2-}$. Because the tips of the $\mathrm{Te} / \mathrm{C}$ nanocables are close (figure 1), it can be concluded that the carbonaceous layers of nanocables have a good permeation capability. $\mathrm{H}^{+}$ ions and $\mathrm{H}_{2} \mathrm{O}_{2}$ molecules can easily be diffused into nanocables. With longer time diffusion and dissolution process, the Te core can be completely consumed. Because this etching was operated at room temperature, these carbonaceous nanotubes kept the original frame of the $\mathrm{Te} / \mathrm{C}$ nanocables. When the chemical etching time was shortened to $45 \mathrm{~min}$, as shown in figure 7 , it could be found that the nanowire core of nanocables was partially etched. The results show that the Te cores are composed of remnants of crude nanowires and discontinuous nanoparticles (figures $7 b, c$ ).

\section{Conclusions}

In conclusion, $\mathrm{Te} / \mathrm{C}$ nanocables have been successfully synthesized by a simple hydrothermal method. The Te/C nanocables are a composite comprising of a smooth core of about $20 \mathrm{~nm}$ in diameter and asurrounding sheath of about $60-80 \mathrm{~nm}$ in thickness. Carbonaceous nanotubes and $\mathrm{Te} / \mathrm{C}$ nanocables with fragmentary Te core were obtained by adjusting time of chemical etching. These carbonaceous nanotubes kept the lengths of the original Te/C nanocables and the sizes of the shell.

\section{Acknowledgement}

The authors gratefully acknowledge the support of Heilongjiang Higher Education Science and Technology Innovation Team Construction Project.

\section{References}

Fang Z, Tang K B, Lei S J and Li T Y 2006 Nanotechnology 17 3008

He Z B and Yu S H 2005 J. Phys. Chem. B109 22740

Hu J Q, Bando Y, Liu Z W, Sekiguchi T, Golberg D and Zhan J H 2003 J. Am. Chem. Soc. 12511306

Jiang X C, Mayers B, Herricks T and Xia Y N 2003 Adv. Mater. 15 1740

Liu Z P, Hu Z K, Xie Q, Yang B J, Wu J and Qian Y T 2003 J. Mater. Chem. 13159

Liu Z P, Hu Z K, Liang J B, Li S, Yang Y, Peng S and Qian Y T 2004 Langmuir 20214

Lu Q Y, Gao F and Komarneni S 2005 Langmuir 216002

Mayers B and Xia Y N 2002 Adv. Mater. 14279

Mo M S, Zeng J H, Liu X M, Yu W C, Zhang S Y and Qian Y T 2002 Adv. Mater. 141658

Song X C, Zhao Y and Zhang Y F 2007a Cryst. Growth Des. 7 159

Song X C, Zheng Y F, Yang E and Wang Y 2007b Mater. Lett. 61 3904

Song X C, Zhao Y, Zheng Y F, Yang E, Chen W Q and Fang Y Q 2008a J. Phys. Chem. C112 5352

Song X C, Zhao Y, Zheng Y F, Yang E, Fu J and He Y 2008b Cryst. Growth Des. 81823

Song X C, Zhao Y, Zheng Y F, Yang E, Chen L Z and Fu F M 2009 Cryst. Growth Des. 9344

Xi G C, Peng Y Y, Yu W C and Qian Y T 2005 Cryst. Growth Des. 5325

Xie Y, Qiao Z P, Chen M, Liu X M and Qian Y T 1999 Adv. Mater. 111512

Zhang M, Bando Y and Wada K 2001 J. Mater. Res. 161408

Zhang Y, Gu H, Suenaga K and Iijima S 1997 Chem. Phys. Lett. 279264 